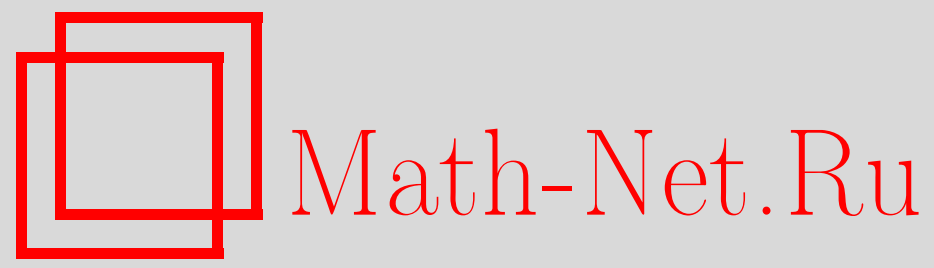

В. Л. Куракин, Семейство последовательностей над кольцом из 8 элементов с низкой корреляцией, Матем. вопр. криптогр., 2010, том 1, выпуск 4, 85-109

DOI: https://doi.org/10.4213/mvk22

Использование Общероссийского математического портала Math-Net.Ru подразумевает, что вы прочитали и согласны с пользовательским соглашением

http://www.mathnet.ru/rus/agreement

Параметры загрузки:

IP : 35.173 .137 .237

26 апреля 2023 г., 10:09:50 
УДК: 519.113.6+512.55

\title{
Семейство последовательностей над кольцом из 8 элементов с низкой корреляцией
}

\author{
В. Л. Куракин \\ Российский государственный социальный университет, г. Москва
}

Получено 20.IV.2010

Строится семейство последовательностей периода $2^{m}-1$ над кольцом из 8 элементов, кросс-корреляционные функции которых асимптотически оптимальны относительно оценок Сидельникова и Уолша.

Ключевые слова: конечные кольца, линейные рекуррентные последовательности, периодические последовательности, кросс-корреляционная функция

A family of sequences over the 8-element ring with low correlation

\section{L. Kurakin}

Russian State Social University, Moscow

Abstract. We construct a family of sequences over the 8-element ring with period $2^{m}-1$ such that their cross-correlation functions are asymptotically optimal with respect to the Sidel'nikov and Welch bounds

Key words: finite rings, linear recurrent sequences, periodic sequences, cross-correlation function

Citation: Mathematical Aspects of Cryptography, 2010, vol. 1, no. 4, pp. 85-109 (Russian). 


\section{1. Корреляционная функция}

Пусть $u$ - последовательность периода $T$ над полем $P=G F(2)$. Kopреляиионная функиия последовательности $u$ определяется равенством

$$
C_{u}(t)=\sum_{i=0}^{T-1}(-1)^{u(i)-u(i+t)}, \quad t \geq 0,
$$

где показатель $u(i)-u(i+t)$ рассматривается не как элемент поля $P$, а как действительное число 0 или 1. Суммирование проводится в поле комплексных чисел $\mathbb{C}$. Так как $C_{u}(t+T)=C_{u}(t)$, часто рассматривают лишь значения $C_{u}(t)$ при $0 \leq t<T$. Значение $C_{u}(0)=T$ и равные ему значения $C_{u}(t)$ при $T \mid t$ называют фазовыми.

Пусть теперь $A-$ некоторое семейство последовательностей над полем $P$, период каждой из которых равен $T$. Кросс-корреляционная функиия последовательностей $u, v \in A$ определяется равенством

$$
C_{u, v}(t)=\sum_{i=0}^{T-1}(-1)^{u(i)-v(i+t)}, \quad t \geq 0 .
$$

Очевидно, $\left|C_{u, v}(t)\right| \leq T$. Справедливо равенство $C_{u}(t)=C_{u, u}(t)$. Значение $C_{u, u}(0)=T$ и равные ему значения $C_{u, u}(t)$ при $T \mid t$ называют фазовыли. Выделяется также значение $C_{u, u+1}(0)=-T$.

Сдвиг последовательности $u$ на $t$ шагов влево обозначим $x^{t} u$, так что

$$
x^{t} u(i)=u(i+t), \quad i \geq 0 .
$$

Тогда кросс-корреляционную функцию можно записать в виде

$$
C_{u, v}(t)=T-2 \rho\left(u, x^{t} v\right),
$$

где $\rho\left(u, x^{t} v\right)=|\{i: 0 \leq i<T, u(i) \neq v(i+t)\}|$ - расстояние Хэмминга между двоичными векторами длины $T$. Поэтому кросс-корреляционную функцию можно воспринимать как меру близости между последовательностями $u$ и $x^{t} v$, которая тем больше по модулю, чем ближе последовательности. Функция $C_{u, v}(t)$ равна 0 тогда и только тогда, когда $u-x^{t} v$ содержит одинаковое количество 0 и 1 на цикле (а значит, $T$ четно).

В системах связи CDMA с разделением доступа каждому пользователю сопоставляется последовательность $u$ из некоторого семейства $A-$ идентификатор пользователя. При вхождении пользователя в связь им посылается 
последовательность $u$, а в центре идентификации принимается последовательность $u$ или ее сдвиг $x^{t} u$. Чтобы надежно (при наличии помех) идентифицировать пользователя, кросс-корреляция между последовательностью $u$ и любой другой последовательностью $v \in A$, а также между сдвигами этих последовательностей должна быть мала - существенно меньше фазового значения $C_{u, u}(0)=T$. Поэтому основным параметром, по которому оцениваются кросс-корреляционные свойства семейства $A$, является число

$$
C_{\max }=C_{\max }(A)=\max \left\{\left|C_{u, v}(t)\right|: u, v \in A, 0 \leq t<T,(u, t) \neq(v, 0)\right\} .
$$

Поскольку пользователь идентифицируется по последовательности $u$ или любому ее сдвигу, семейство $A$ вместе с любой последовательностью должно содержать все ее сдвиги. Пользователю при этом сопоставляется цикл - множество всех сдвигов последовательности $u$. Поэтому наряду с семейством $A$, замкнутым относительно сдвигов, мы будем рассматривать семейство $\widetilde{A}$, которое состоит из выбранных каким-то образом представителей всех циклов семейства $A$. Очевидно, $|\widetilde{A}|=|A| / T$.

Понятие кросс-корреляционной функции обобщается на недвоичные последовательности следующим образом. Пусть $\mathbb{Z}_{m}-$ кольцо вычетов, $\omega \in \mathbb{C}-$ первообразный корень степени $m$ из 1 в поле комплексных чисел. Отображение

$$
\chi: \mathbb{Z}_{m} \rightarrow \mathbb{C}^{*}, \quad \chi(a)=\omega^{a}=e^{2 \pi i a}, \quad 0 \leq a<m,
$$

является характером группы $\left(\mathbb{Z}_{m},+\right)$, т. е. аддитивным характером кольия $\mathbb{Z}_{m}$. Пусть $A$ - семейство последовательностей периода $T$ над кольцом $\mathbb{Z}_{m}$. Кросс-корреляционная функция последовательностей $u, v \in A$ определяется равенством

$$
C_{u, v}(t)=\sum_{i=0}^{T-1} \chi(u(i)-v(i+t))=\sum_{i=0}^{T-1} \omega^{u(i)-v(i+t)}, \quad t \geq 0 .
$$

Коррелящионная функиия последовательности $u$ по определению равна $C_{u}(t)=C_{u, u}(t)$. Очевидно, $\left|C_{u, v}(t)\right| \leq T$. Значения $C_{u, u}(0)=C_{u}(0)=T$ называют фазовыми. Параметр $C_{\max }$ определяется так же, как и выше.

Представляют интерес семейства с возможно меньшим значением параметра $C_{\max }$. Существуют теоретические нижние оценки для этого параметра, наиболее известные из которых - оценки Сидельникова и Уолша. Согласно оиенке Сидельникова [6], для семейства из $|A|=T+1$ последовательностей периода $T$ параметр $C_{\max }$ при больших $T$ ограничен снизу:

$$
C_{\max } \geq \sqrt{\alpha T},
$$


где $\alpha=2$ для двоичных и $\alpha=1$ для недвоичных последовательностей. Первое семейство $p$-ичных последовательностей ( $p \geq 3$ простое), для которых достигается эта оценка, было построено В. М. Сидельниковым. Из оценки (1) видно, что в недвоичном случае теоретически возможно строить семейства последовательностей, для которых параметр $C_{\max }$ будет в $\sqrt{2}$ раз меньше, чем в двоичном случае. Это объясняет интерес к недвоичным последовательностям.

Оиенка Уолиа [10] (в одной из ее формулировок) утверждает, что для семейства из $|A|=M$ последовательностей периода $T$ верно неравенство

$$
C_{\max }^{2} \geq \frac{T^{2}(M-1)}{M T-1} .
$$

При $M \rightarrow \infty$ оценка показывает, что асимптотически $C_{\max } \geq \sqrt{T}$. Семейства, для которых это неравенство асимптотически обращается в равенство, т.е. $C_{\max } \sim \sqrt{T}$ при $M \rightarrow \infty$, называют асимптотически оптимальными относительно оценки Уолша.

\section{2. Пример оптимальных семейств последовательностей над $\mathbb{Z}_{4}$}

К настоящему времени описано довольно много семейств последовательностей, асимптотически оптимальных относительно оценок Сидельникова и Уолша. Мы рассмотрим семейства из работы [7], поскольку семейство, которое строится в данной работе, является их обобщением.

В дальнейшем через $G R\left(q^{n}, p^{n}\right)$ будем обозначать кольцо Галуа характеристики $p^{n}$, состоящее из $q^{n}$ элементов $[4,8,9]$. Пусть $R=\mathbb{Z}_{4}=G R(4,4)-$ кольцо вычетов по модулю 4 и $R^{\prime}=G R\left(4^{m}, 4\right)$ - его расширение Галуа степени $m$. Обозначим через

$$
\Gamma\left(R^{\prime}\right)=\left\{b \in R^{\prime}: b^{2^{m}}=b\right\}
$$

2-адическое координатное множество кольца $R^{\prime}$. Это множество состоит из $2^{m}$ элементов, попарно различных по модулю радикала $2 R^{\prime}$ кольца $R^{\prime}$, и замкнуто относительно умножения. Любой элемент $a \in R^{\prime}$ однозначно представляется в виде

$$
a=a_{0}+2 a_{1}, \quad \text { где } a_{0}=\gamma_{0}(a), a_{1}=\gamma_{1}(a) \in \Gamma\left(R^{\prime}\right),
$$

который называется 2-адическим разложением элемента $a$. Относительно операций сложения $a \oplus b=\gamma_{0}(a+b), a, b \in \Gamma\left(R^{\prime}\right)$, и умножения кольца $R^{\prime}$ множество $\Gamma\left(R^{\prime}\right)$ образует поле $G F\left(2^{m}\right)$. 
Пусть $F(x) \in R[x]$ - такой унитарный многочлен степени $m$, что его образ $\bar{F}(x)$ над полем вычетов $\bar{R}=R / 2 R=G F(2)$ неприводим над полем $\bar{R}$. Такой многочлен называется многочленом Галуа. В дальнейшем предполагается, что период $T(\bar{F})$ многочлена $\bar{F}(x)$ равен $T(\bar{F})=2^{m}-1$, т. е. $\bar{F}(x)$ есть многочлен максимального периода над полем вычетов $\bar{R}$. Тогда период $T(F)$ многочлена $F(x)$ над кольцом $R$ может быть равен $2^{m}-1$ или $2\left(2^{m}-1\right)$. В первом случае многочлен $F(x)$ называется отмеченным, во втором - многочленом максимального периода (ММП) над кольцом $R$. В кольце Галуа $R^{\prime}=G R\left(4^{m}, 4\right)$ многочлен $F(x)$ имеет корень $\alpha$. Многочлен $F(x)$ отмеченный тогда и только тогда, когда $\alpha \in \Gamma\left(R^{\prime}\right)$, т. е. $\alpha=\gamma_{0}(\alpha)$.

Обозначим через $L_{R}(F)$ множество всех линейных рекуррентых последовательностей (ЛРП) над кольцом $R$ с характеристическим многочленом $F(x)$. Тогда

$$
L_{R}^{*}(F)=\left\{u \in L_{R}(F): \bar{u} \neq 0\right\}=L_{R}(F) \backslash 2 L_{R}(F)
$$

есть множество последовательностей из $L_{P}(F)$, у которых начальный вектор не равен нулевому вектору по модулю 2 .

В работе [7] рассматриваются два семейства последовательностей над кольцом $R=\mathbb{Z}_{4}$, которые мы будем обозначать $A_{0}$ и $B_{0}$ (в [7] они обозначаются $A$ и $B$ ). Эти семейства определяются следующим образом:

$$
\begin{array}{ll}
A_{0}=L_{R}(F) \backslash 0, & \text { где } F(x) \text { - отмеченный многочлен, } \\
B_{0}=L_{R}^{*}(F), & \text { где } F(x)-\text { ММП. }
\end{array}
$$

Причем во втором случае на корень $\alpha$ многочлена $F(x)$ накладываются условия, формулируемые в терминах p-адического разложения (3) следующим образом: если

$$
\alpha=\alpha_{0}+2 \alpha_{1}=\alpha_{0}(1+2 \delta), \quad \text { где } \delta=\alpha_{0}^{-1} \alpha_{1},
$$

то

$$
\delta \neq 1 \text { и } \operatorname{tr}_{1}^{m}(\bar{\delta}) \neq 1
$$

Здесь

$$
\operatorname{tr}=\operatorname{tr}_{1}^{m}=\operatorname{tr}_{G F(2)}^{G F\left(2^{m}\right)}=\operatorname{tr}_{\Gamma(R)}^{\Gamma\left(R^{\prime}\right)}
$$

- след из поля $\Gamma\left(R^{\prime}\right)=G F\left(2^{m}\right)$ в поле $\Gamma(R)=G F(2)$, определяемый равенством

$$
\operatorname{tr}(x)=x \oplus x^{2} \oplus x^{4} \oplus \ldots \oplus x^{2^{m-1}} .
$$

$\mathrm{B}$ следующей таблице перечислены параметры семейств $A_{0}$ и $B_{0}$ : число последовательностей $M$, период последовательностей $T$, число циклов $M / T$ 
и верхняя оценка кросс-корреляции последовательностей $C_{\max }$, доказанная в [7].

\begin{tabular}{|c|c|c|c|c|}
\hline & $M$ & $T$ & $M / T$ & $C_{\max } \leq$ \\
\hline$A_{0}$ & $2^{2 m}-1$ & $2^{m}-1$ & $2^{m}+1=T+2$ & $1+\sqrt{T+1}$ \\
\hline$B_{0}$ & $2^{2 m}-2^{m}$ & $2\left(2^{m}-1\right)$ & $2^{m-1}=\frac{T+2}{4}$ & $2+\sqrt{T+2}$ \\
\hline
\end{tabular}

Таким образом, семейства асимптотически оптимальны относительно оценок Сидельникова и Уолша.

Последовательности семейств $A_{0}$ и $B_{0}$ имеют представление функцией след. Напомним, что функция след из кольца Галуа $R^{\prime}$ в кольцо $R$ определяется равенством

$$
\operatorname{Tr}(a)=\operatorname{Tr}_{R}^{R^{\prime}}(a)=\sum_{\varphi \in \operatorname{Aut}\left(R^{\prime} / R\right)} \varphi(a), \quad a \in R^{\prime} .
$$

Каждая последовательность $u \in A_{0}$ представляется в виде

$$
u(i)=\operatorname{Tr}_{R}^{R^{\prime}}\left(c \alpha^{i}\right), \quad c \neq 0, \quad \text { где } \alpha=\alpha_{0} \in \Gamma\left(R^{\prime}\right) .
$$

В данной работе строится семейство $A$ последовательностей над кольцом из 8 элементов, являющееся аналогом семейства $A_{0}$.

Функцию след над кольцом Галуа можно записать в явном виде, используя $p$-адическое разложение элементов. Если $a \in \Gamma\left(R^{\prime}\right)$, то

$$
\operatorname{Tr}_{R}^{R^{\prime}}(a)=\sum_{r=0}^{m-1} a^{2^{r}}, \quad a \in \Gamma\left(R^{\prime}\right) .
$$

Поэтому для произвольного элемента $a \in R^{\prime}$ с $p$-адическим разложением $a=a_{0}+2 a_{1}$ справедливо равенство [4]

$$
\operatorname{Tr}_{R}^{R^{\prime}}(a)=\operatorname{Tr}\left(a_{0}\right)+2 \operatorname{Tr}\left(a_{1}\right)=\sum_{r=0}^{m-1}\left(a_{0}^{2^{r}}+2 a_{1}^{2^{r}}\right), \quad a \in R^{\prime} .
$$

\section{3. Последовательности над кольцом $S$ из 8 элементов}

Пусть по-прежнему $R=\mathbb{Z}_{4}$. Рассмотрим кольцо $S=R[x] /\left(x^{2}, 2 x\right)$, состоящее из 8 элементов. Согласно [5] $S$ является наименьшим неквазифробениусовым кольцом (существует еще одно такое кольцо $\mathbb{Z}_{2}[x, y] /\left(x^{2}, x y, y^{2}\right)$, но оно представляет меньший интерес, так как имеет характеристику 2). 
Последовательности над кольцом $S$, их представление функцией след, ранги координатных последовательностей и частотные характеристики рассматривались в [1-3]. В этой работе строится семейство последовательностей над кольцом $S$ с низкой кросс-корреляцией.

Перечислим свойства кольца $S$, указанные в [2]. Пусть $\theta \in S-$ образ элемента $x$ при естественном эпиморфизме $R[x] \rightarrow S$ с ядром $\left(x^{2}, 2 x\right)$. Тогда $\theta^{2}=2 \theta=0$ и

$$
S=R[\theta]=\{a+b \theta: a \in R, b \in\{0,1\}\}=\{0,1,2,3, \theta, 1+\theta, 2+\theta, 3+\theta\} .
$$

Кольцо $S$ локально, его максимальный идеал, совпадающий с радикалом, равен

$$
J=J(S)=(2, \theta)=\{0,2, \theta, 2+\theta\},
$$

а поле вычетов есть

$$
\bar{S}=S / J(S)=G F(2) .
$$

Так как $J^{2}=0$, то индекс нильпотентности радикала $J$ равен 2. Если $a \in S$, $F(x) \in S[x], u \in S^{\infty}$ - соответственно элемент, многочлен и последовательность над кольцом $S$, то через $\bar{a}, \bar{F}(x), \bar{u}$ будем обозначать их образы при естественном эпиморфизме $S \rightarrow \bar{S}$ кольца $S$ в его поле вычетов при индуцируемыми им эпиморфизмами $S[x] \rightarrow \bar{S}[x]$ и $S^{\infty} \rightarrow \bar{S}^{\infty}$. Унитарный многочлен $F(x) \in S[x]$ такой, что $\bar{F}(x)$ неприводим над полем $\bar{S}$, будем называть многочленом Галуа над кольцом $S$.

Как видно из (6), аддитивная группа $(S,+)$ кольца $S$ есть прямая сумма

$$
(S,+)=\langle 1\rangle \dot{+}\langle\theta\rangle \cong \mathbb{Z}_{4} \oplus \mathbb{Z}_{2} .
$$

Соответственно, мультипликативная группа $S^{\wedge}$ аддитивных характеров кольца $S$ имеет вид

$$
\left(S^{\wedge}, \cdot\right)=\left\langle\chi_{1}\right\rangle \dot{x}\left\langle\chi_{2}\right\rangle,
$$

где характеры $\chi_{1}, \chi_{2}: S \rightarrow \mathbb{C}$ определяются равенствами

$$
\chi_{1}(a+b \theta)=\omega^{a}, \quad \chi_{2}(a+b \theta)=(-1)^{b}, \quad \omega=\sqrt{-1} .
$$

Здесь $a \in R=\{0,1,2,3\}, b \in\{0,1\}$. Рассмотрим характер $\chi=\chi_{1} \chi_{2}$. Тогда

$$
\chi(a+b \theta)=\omega^{a}(-1)^{b}=\omega^{a+2 b} .
$$

Пусть $u, v-$ две последовательности периода $T$ над кольцом $S$. Кросскорреляционную функциию последовательностей $u, v$ определим равенством

$$
C_{u, v}(t)=\sum_{i=0}^{T-1} \chi(u(i)-v(i+t)), \quad t \geq 0 .
$$

Очевидно, $\left|C_{u, v}(t)\right| \leq T$ и $C_{u, u}(0)=T$. 
Для произвольного унитарного реверсивного (т. е. $F(0) \in S^{*}$ ) многочлена $F(x) \in S[x]$ степени $m$ и ЛРП $u \in L_{S}(F)$ верны оценки $T(u) \leq T(F) \leq$ $\leq 2\left(2^{m}-1\right)$, аналогичные оценкам для многочленов над кольцом Галуа. Если $T(F)=2\left(2^{m}-1\right)$, то многочлен $F(x)$ назовем многочленом максимального периода над кольиом $S$, а ЛРП и ранга $m$ и периода $2\left(2^{m}-1\right)$ назовем линейной рекуррентной последовательностью максимального периода (ЛРП МП) над кольцом $S$. Если $T(\bar{F})=T(F)=2^{m}-1$, то многочлен $F(x)$ назовем отмеченным многочленом над кольиом $S$.

Если $F(x)$ - отмеченный многочлен, то все ненулевые последовательности из $L_{S}(F)$ имеют период $2^{m}-1$. Пусть $F(x)-$ многочлен максимального периода. Обозначим

$$
L_{S}^{*}(F)=\left\{u \in L_{S}(F): \bar{u} \neq 0\right\}=L_{S}(F) \backslash\left(2 L_{S}(F)+\theta L_{S}(F)\right) .
$$

Тогда все последовательности из $L_{S}^{*}(F)$ имеют период $2\left(2^{m}-1\right)$, а остальные ненулевые последовательности из $L_{S}(F)-$ период $2^{m}-1$, (см. [2, утв. 3.13]).

Над кольцом $S$ можно определить семейства последовательностей, аналогичные семействам $A_{0}, B_{0}$ из [7]. Пусть $F(x) \in S[x]-$ такой многочлен Галуа степени $m \geq 1$, что $\bar{F}(x)-$ многочлен максимального периода над полем $\bar{S}$. Тогда $F(x)$ - либо отмеченный многочлен, либо многочлен максимального периода. Если многочлен $F(x)$ отмеченный, то рассмотрим семейство

$$
A=L_{S}(F) \backslash 0 .
$$

Это семейство состоит из $|S|^{m}-1=2^{3 m}-1$ последовательностей периода $2^{m}-1$ каждая. Если $F(x)$ - многочлен максимального периода, то рассмотрим семейство

$$
B=L_{S}^{*}(F) .
$$

Оно состоит из $2^{3 m}-2^{2 m}$ последовательностей периода $2\left(2^{m}-1\right)$.

Многочлен $F(x) \in S[x]$ можно выбрать так, чтобы выполнялось включение $F(x) \in R[x]$. Тогда семейства $A_{0}, B_{0}$ будут подмножествами в $A$ и $B$. В общем случае это не так.

$\mathrm{B}$ данной работе изучается семейство $A$. В следующей таблице для сравнения перечислены параметры семейств $A_{0}, B_{0}, A$ и $B$ : мощность алфавита $m=|R|$ или $m=|S|$, число последовательностей $M$, период последовательностей $T$, число циклов $M / T$ и верхняя оценка кросс-корреляции $C_{\max }$, которая для семейства $A$ будет доказана ниже. Информация о семействах $A_{0}$ и $B_{0}$ из [7] приведена в таблице для удобства их сравнения с семействами $A$ и $B$. 


\begin{tabular}{|c|c|c|c|c|c|}
\hline & $q$ & $M$ & $T$ & $M / T$ & $C_{\max } \leq$ \\
\hline$A_{0}$ & 4 & $2^{2 m}-1$ & $2^{m}-1$ & $2^{m}+1$ & $1+\sqrt{T+1}$ \\
\hline$B_{0}$ & 4 & $2^{2 m}-2^{m}$ & $2\left(2^{m}-1\right)$ & $2^{m-1}$ & $2+\sqrt{T+2}$ \\
\hline$A$ & 8 & $2^{3 m}-1$ & $2^{m}-1$ & $2^{2 m}+2^{m}+1$ & $1+\sqrt{T+1}$ \\
\hline$B$ & 8 & $2^{3 m}-2^{2 m}$ & $2\left(2^{m}-1\right)$ & $2^{2 m-1}$ & \\
\hline
\end{tabular}

Из таблицы видно, что семейство $A$ асимптотически оптимально относительно оценок Сидельникова и Уолша. По сравнению с семейством $A_{0}$, последовательности из $A$ имеют те же периоды и оценки кросс-корреляции, но число последовательностей в семействе $A$ больше, чем в $A_{0}$. Здесь следует учитывать изменение размера алфавита: последовательности в семействах $A_{0}$ и $B_{0}$ четверичные, а в семействах $A$ и $B$ - восьмеричные. Именно за счет этого и происходит увеличение числа последовательностей в семействах $A$ и $B$.

\section{4. Функция след над кольцом $S$}

Следующие результаты доказаны в [2].

Пусть $R^{\prime}=G R\left(4^{m}, 4\right)-$ расширение Галуа степени $m$ (см. [4, 8, 9]) кольца Галуа $R=\mathbb{Z}_{4}=G R(4,4)$. Рассмотрим факторкольцо $S^{\prime}=$ $=R^{\prime}[x] /\left(x^{2}, 2 x\right)$. Обозначим через $\theta \in S^{\prime}$ образ элемента $x$ при естественном эпиморфизме $R^{\prime}[x] \rightarrow S^{\prime}$ с ядром $\left(x^{2}, 2 x\right)$. Тогда $\theta^{2}=2 \theta=0$, кольцо $S^{\prime}$ является расширением $S^{\prime}=R^{\prime}[\theta]$ кольца $R^{\prime}$ элементом $\theta$, а кольцо $S$ естественным образом отождествляется с подкольцом $R[\theta]$ кольца $S^{\prime}$. Таким образом,

$$
R \subset S \subset S^{\prime}, \quad R \subset R^{\prime} \subset S^{\prime}, \quad S=R[\theta], \quad S^{\prime}=R^{\prime}[\theta] .
$$

Кольцо $S^{\prime}$ локально, его максимальный идеал, совпадающий с радикалом, равен

$$
J^{\prime}=J\left(S^{\prime}\right)=(2, \theta)=\left\{2 a+b \theta: a, b \in R^{\prime}\right\}
$$

и имеет индекс нильпотентности 2. Порядки и поля вычетов рассматриваемых колец следующие:

$$
\begin{gathered}
|R|=4, \quad|S|=8, \quad\left|R^{\prime}\right|=4^{m}, \quad\left|S^{\prime}\right|=8^{m}, \\
\bar{R}=R / 2 R \cong \bar{S}=S / J=G F(2), \quad \bar{R}^{\prime}=R^{\prime} / 2 R^{\prime} \cong \bar{S}^{\prime}=S^{\prime} / J^{\prime}=G F\left(2^{m}\right) .
\end{gathered}
$$

В дальнейшем через $\bar{a}, \bar{F}(x)$ и $\bar{u}$ будем обозначать образ элемента $a \in S^{\prime}$, многочлена $F(x) \in S^{\prime}[x]$ и последовательности $u \in\left(S^{\prime}\right)^{\infty}$ под действием естественных эпиморфизмов $S^{\prime} \rightarrow \bar{S}^{\prime}, S^{\prime}[x] \rightarrow \bar{S}^{\prime}[x]$ и $\left(S^{\prime}\right)^{\infty} \rightarrow\left(\bar{S}^{\prime}\right)^{\infty}$ соответственно. Элементы кольца $S$ имеют вид

$$
S^{\prime}=\left\{a+b \theta: a, b \in R^{\prime}\right\}
$$


причем $a+b \theta=c+d \theta, a, b, c, d \in R^{\prime}$, тогда и только тогда, когда $a=c$, $\bar{b}=\bar{d}$.

Функция след из кольца $S^{\prime}$ в $S$ определяется равенством

$$
\operatorname{Tr}(a)=\operatorname{Tr}_{S}^{S^{\prime}}(a)=\sum_{\varphi \in \operatorname{Aut}\left(S^{\prime} / S\right)} \varphi(a), \quad a \in S^{\prime}
$$

Автоморфизмы кольца $S^{\prime}$ над $S$, а значит, и функцию след можно описать явно, для этого требуется ввести 2-адическое разложение для элементов кольца $S^{\prime}$.

Множество $\Gamma\left(S^{\prime}\right)=\left\{b \in S^{\prime}: b^{2^{m}}=b\right\}$ назовем 2-адическим координатным множеством кольц̧а $S^{\prime}$. Справедливо равенство $\Gamma\left(S^{\prime}\right)=\Gamma\left(R^{\prime}\right)$. В частности, при $m=1$ получаем $\Gamma(S)=\Gamma(R)=\{0,1\}$.

Каждый элемент $a \in S^{\prime}$ однозначно представляется в виде

$$
a=a_{0}+2 a_{1}+\theta a_{\theta}, \quad a_{0}, a_{1}, a_{\theta} \in \Gamma\left(S^{\prime}\right) .
$$

Элементы $a_{0}, a_{1}, a_{\theta}$ назовем 2-адическими координатами элемента а и будем обозначать следующим образом:

$$
a_{0}=\gamma_{0}(a), \quad a_{1}=\gamma_{1}(a), \quad a_{\theta}=\gamma_{\theta}(a), \quad a \in S^{\prime}
$$

Координату $a_{0}$ будем называть нулевой, или младшей, а координаты $a_{1}, a_{\theta}-$ первыми, или стариими. Легко увидеть, что алгебра $\left(\Gamma\left(S^{\prime}\right), \oplus, \cdot\right)$, где $a \oplus b=$ $=\gamma_{0}(a+b), a, b \in S^{\prime}$, есть поле, изоморфное полю $\bar{S}^{\prime}=G F\left(2^{m}\right)$. Изоморфизм $\Gamma\left(S^{\prime}\right) \rightarrow \bar{S}^{\prime}$ задается соотношениями $a \rightarrow \bar{a}, a \in \Gamma\left(S^{\prime}\right)$. Для произвольного элемента $a \in S^{\prime}$ выполняется равенство $\gamma_{0}(a)=a^{2^{m}}$.

Группа автоморфизмов $\operatorname{Aut}\left(S^{\prime} / S\right)$ кольца $S^{\prime}$ над $S$ является циклической группой порядка $m$ и порождается автоморфизмом Фробениуса $\rho$, действующим по правилу

$$
\rho(a)=\gamma_{0}(a)^{2}+2 \gamma_{1}(a)^{2}+\theta \gamma_{\theta}(a)^{2}, \quad a \in S^{\prime} .
$$

Отсюда, а также из соотношения (5) вытекает следующее явное описание функции след с использованием $p$-адического разложения элементов кольца $S^{\prime}$ :

$$
\begin{aligned}
\operatorname{Tr}_{S}^{S^{\prime}}(a) & =\operatorname{Tr}\left(a_{0}\right)+2 \operatorname{Tr}\left(a_{1}\right)+\theta \operatorname{Tr}\left(a_{\theta}\right)= \\
& =\sum_{r=0}^{m-1}\left(a_{0}^{2^{r}}+2 a_{1}^{2^{r}}+\theta a_{\theta}^{2^{r}}\right), \quad a \in S^{\prime}
\end{aligned}
$$


Отображение $\operatorname{Tr}_{S}^{S^{\prime}}$ является сюръективным отображением множества $S^{\prime}$ на $S$, линейным над $S$, и его ограничение на множество $R^{\prime}$ совпадает с функцией след $\operatorname{Tr}_{R}^{R^{\prime}}$.

Опишем представление последовательностей семейств $A$ и $B$ с помощью функции след.

Пусть $F(x) \in S[x]-$ реверсивный многочлен Галуа степени $m$. В кольце $S^{\prime}$ многочлен $F(x)$ имеет корень $\alpha$, и $\alpha, \rho(\alpha), \ldots, \rho^{m-1}(\alpha)$ - все его корни в $S^{\prime}$. Если к тому же $T(\bar{F})=2^{m}-1$, то многочлен $F(x)$ является отмеченным тогда и только тогда, когда $\alpha=\gamma_{0}(\alpha)$, т. е. $\alpha \in \Gamma\left(S^{\prime}\right)$. В противном случае, т. е. при $\alpha \neq \gamma_{0}(\alpha), F(x)$ есть многочлен максимального периода.

Для любой ЛРП $u \in L_{S}(F)$ существует единственный элемент $c \in S^{\prime}$, для которого

$$
u(i)=\operatorname{Tr}_{S}^{S^{\prime}}\left(c \alpha^{i}\right), \quad i \geq 0 .
$$

В частности, такое представление имеют последовательности семейств $A$ и $B$, причем включения $u \in A, u \in B$ равносильны условиям $c \neq 0$ и $\bar{c} \neq 0$ соответственно.

Как известно, функция след $\operatorname{tr}=\operatorname{tr}_{q}^{q^{m}}$ над полем удовлетворяет соотношениям ортогональности

$$
\sum_{x \in G F\left(q^{m}\right)}(-1)^{\operatorname{tr}(x)}=0, \quad \sum_{x \in G F\left(q^{m}\right)^{*}}(-1)^{\operatorname{tr}(x)}=-1 .
$$

Аналогичные соотношения для функции след над кольцом Галуа приведены в [7, с. 1104]. В дальнейшем потребуются следующие соотношения ортогональности для функции след над кольцом $S$.

Лемма 1. Пусть $\operatorname{Tr}=\operatorname{Tr}_{S}^{S^{\prime}} u \chi-$ характер кольиа $S$, определенный в (7). Тогда:
a) $\sum_{x \in S^{\prime}} \chi(\operatorname{Tr}(x))=0$
б) $\sum_{x \in J\left(S^{\prime}\right)} \chi(\operatorname{Tr}(x))=0$;
в) $\sum_{x \in\left(S^{\prime}\right)^{*}} \chi(\operatorname{Tr}(x))=0$;
г) $\sum_{x \in\left(R^{\prime}\right)^{*}} \chi(\operatorname{Tr}(c x))=0$ для любого $c \in\left(S^{\prime}\right)^{*}$. 
Доказательство. а) Отображение $\operatorname{Tr}: S^{\prime} \rightarrow S$ линейно над $S$ и сюръективно, поэтому каждый элемент $a \in S$ имеет одинаковое число прообразов: $\left|\operatorname{Tr}^{-1}(a)\right|=\left|S^{\prime}\right| /|S|=2^{3 m-3}$. Поэтому

$$
\sum_{x \in S^{\prime}} \chi(\operatorname{Tr}(x))=2^{3 m-3} \sum_{a \in S} \chi(a)=0,
$$

где последнее равенство есть соотношение ортогональности для характера $\chi$ группы $(S,+)$.

б) Элементы $x \in J\left(S^{\prime}\right)$ имеют вид $x=2 x_{1}+\theta x_{\theta}$. Поэтому искомая сумма равна

$$
\begin{gathered}
\sum_{x_{1}, x_{\theta} \in \Gamma\left(S^{\prime}\right)} \chi\left(\operatorname{Tr}\left(2 x_{1}+\theta x_{\theta}\right)\right)=\sum_{x_{1}, x_{\theta} \in \Gamma\left(S^{\prime}\right)} \chi\left(2 \operatorname{tr}\left(x_{1}\right)+\theta \operatorname{tr}\left(x_{\theta}\right)\right)= \\
=\sum_{x_{1}, x_{\theta} \in \Gamma\left(S^{\prime}\right)}(-1)^{\operatorname{tr}\left(x_{1}\right)}(-1)^{\operatorname{tr}\left(x_{\theta}\right)}=\sum_{x_{1} \in \Gamma\left(S^{\prime}\right)}(-1)^{\operatorname{tr}\left(x_{1}\right)} \cdot \sum_{x_{\theta} \in \Gamma\left(S^{\prime}\right)}(-1)^{\operatorname{tr}\left(x_{\theta}\right)}=0 \cdot 0=0 .
\end{gathered}
$$

в) Так как $\left(S^{\prime}\right)^{*}=S \backslash J\left(S^{\prime}\right)$, то искомая сумма равна разности сумм из а) и б).

г) Представим элементы $c, x$ в виде $c=a+b \theta, x=y(1+2 z)$, где $a \in R^{\prime}, b, y, z \in \Gamma\left(R^{\prime}\right)$, причем $\bar{a} \neq \overline{0}, y \neq 0$. Тогда

$$
\begin{gathered}
\operatorname{Tr}(c x)=\operatorname{Tr}((a+b \theta) y(1+2 z))= \\
=\operatorname{Tr}(a y+2 a y z+b y \theta)=\operatorname{Tr}(a y)+2 \operatorname{tr}(a y z)+\theta \operatorname{tr}(b y) .
\end{gathered}
$$

Искомая сумма равна

$$
\begin{gathered}
\sum_{\substack{y, z \in \Gamma\left(R^{\prime}\right) \\
y \neq 0}} \chi(\operatorname{Tr}(a y)+2 \operatorname{tr}(a y z)+\theta \operatorname{tr}(b y))= \\
=\sum_{y \in \Gamma\left(R^{\prime}\right)^{*}} \chi(\operatorname{Tr}(a y)+\theta \operatorname{tr}(b y)) \sum_{z \in \Gamma\left(R^{\prime}\right)} \chi(2 \operatorname{tr}(a y z)) .
\end{gathered}
$$

Здесь внутренняя сумма по $z$ равна

$$
\sum_{\bar{z} \in \bar{R}^{\prime}}(-1)^{\operatorname{tr}(\overline{a y z})}=\sum_{\bar{z} \in \bar{R}^{\prime}}(-1)^{\operatorname{tr}(\bar{z})}=0,
$$

где первое равенство следует из того, что $\overline{a y} \neq 0$, а второе - из соотношения ортогональности для функции след над полем. Следовательно, и искомая сумма равна 0 . 


\section{5. Вычисление тригонометрической суммы над кольцом $S$}

Аналогично [7], для исследования кросс-корреляционной функции последовательностей семейства $A$ рассмотрим тригонометрическую сумму над кольцом $S$ :

$$
\zeta(\gamma)=\sum_{x \in \Gamma\left(S^{\prime}\right)^{*}} \chi(\operatorname{Tr}(\gamma x)), \quad \gamma \in S^{\prime}
$$

где $\chi: S \rightarrow \mathbb{C}-$ характер, определенный в (7), и $\operatorname{Tr}=\operatorname{Tr}_{S}^{S^{\prime}}$. Кросскорреляционная функция любых двух последовательностей $u, u^{\prime} \in A$ равна $\zeta(\gamma)$ для некоторого $\gamma$. Действительно, используя представление (12), запишем

$$
u(i)=\operatorname{Tr}\left(c \alpha^{i}\right), \quad u^{\prime}(i)=\operatorname{Tr}\left(c^{\prime} \alpha^{i}\right), \quad i \geq 0,
$$

где $c, c^{\prime} \in S^{\prime} \backslash 0$ и $\alpha$ - корень отмеченного многочлена $F(x)$ степени $m$ и периода $T=2^{m}-1$. Тогда

$$
C_{u, u^{\prime}}(t)=\sum_{i=0}^{T-1} \chi\left(\operatorname{Tr}\left(c \alpha^{i}\right)-\operatorname{Tr}\left(c^{\prime} \alpha^{i+t}\right)\right)=\sum_{i=0}^{T-1} \chi\left(\operatorname{Tr}\left(\left(c-c^{\prime} \alpha^{t}\right) \alpha^{i}\right)\right) .
$$

Так как многочлен $F(x)$ отмеченный, то $\alpha \in \Gamma\left(S^{\prime}\right)$, значит, $\alpha^{i}$ пробегает множество $\Gamma\left(S^{\prime}\right)^{*}$. Поэтому

$$
C_{u, u^{\prime}}(t)=\zeta(\gamma), \quad \text { где } \gamma=c-c^{\prime} \alpha^{t} .
$$

Следовательно, для нахождения значений кросс-корреляционной функции последовательностей из $A$ достаточно найти значения суммы $\zeta(\gamma)$.

Утверждение 2. Если $\gamma \in\left(S^{\prime}\right)^{*}$, mo $|1+\zeta(\gamma)|^{2}=2^{m}$.

Доказательство. По определению $\zeta(\gamma)$

$$
\begin{aligned}
|\zeta(\gamma)|^{2} & =\zeta(\gamma) \bar{\zeta}(\gamma)=\sum_{x \in \Gamma\left(S^{\prime}\right)^{*}} \chi(\operatorname{Tr}(\gamma x)) \cdot \sum_{y \in \Gamma\left(S^{\prime}\right)^{*}} \bar{\chi}(\operatorname{Tr}(\gamma y))= \\
& =\sum_{x, y \in \Gamma\left(S^{\prime}\right)^{*}} \chi(\operatorname{Tr}(\gamma x)) \chi(-\operatorname{Tr}(\gamma y))=\sum_{x, y \in \Gamma\left(S^{\prime}\right)^{*}} \chi(\operatorname{Tr}(\gamma(x-y)))= \\
& =\sum_{x, y \in \Gamma\left(S^{\prime}\right)^{*}} \chi\left(\operatorname{Tr}\left(\gamma x\left(1-\frac{y}{x}\right)\right),\right.
\end{aligned}
$$


где черта над комплексным числом означает комплексное сопряжение. Пусть $x \in \Gamma\left(S^{\prime}\right)^{*}$ фиксирован, а $y$ пробегает множество $\Gamma\left(S^{\prime}\right)^{*}=\Gamma\left(R^{\prime}\right)^{*}$. Тогда частное $y / x$ пробегает в точности множество $\Gamma\left(S^{\prime}\right)^{*}$. В силу [7, лемма 1 , c. 1105] разность $1-\frac{y}{x}$ пробегает в точности по одному разу нулевое значение $($ при $y=x$ ) и множество представителей всех различных смежных классов мультипликативной группы $\left(R^{\prime}\right)^{*}$ по подгруппе $\Gamma\left(R^{\prime}\right)^{*}$, кроме представителей двух классов $\pm \Gamma\left(R^{\prime}\right)^{*}$. Отсюда следует, что разность

$$
z=x-y=x\left(1-\frac{y}{x}\right), \quad x, y \in \Gamma\left(S^{\prime}\right)^{*},
$$

$\left|\Gamma\left(S^{\prime}\right)^{*}\right|=2^{m}-1$ раз принимает значение 0 при $y=x$, а при $y \neq x$ принимает в точности по одному разу все значения из $\left(R^{\prime}\right)^{*} \backslash\left( \pm \Gamma\left(R^{\prime}\right)^{*}\right)$. Отсюда получаем:

$$
\begin{aligned}
|\zeta(\gamma)|^{2}= & \left(2^{m}-1\right) \chi(\operatorname{Tr}(0))+\sum_{z \in\left(R^{\prime}\right)^{*}} \chi(\operatorname{Tr}(\gamma z))- \\
& -\sum_{z \in \Gamma\left(R^{\prime}\right)^{*}} \chi(\operatorname{Tr}(\gamma z))-\sum_{z \in \Gamma\left(R^{\prime}\right)^{*}} \chi(\operatorname{Tr}(-\gamma z)) .
\end{aligned}
$$

Здесь $\chi(\operatorname{Tr}(0))=\chi(0)=1$, первая сумма равна 0 по лемме 1г), а вторая и третья суммы равны $\zeta(\gamma)$ и $\bar{\zeta}(\gamma)$ по определению (14). В итоге

$$
|\zeta(\gamma)|^{2}=2^{m}-1-\zeta(\gamma)-\bar{\zeta}(\gamma) .
$$

Отсюда $|1+\zeta(\gamma)|^{2}=(1+\zeta(\gamma))(1+\bar{\zeta}(\gamma))=2^{m}$.

Теорема 3. Пусть элемент $\gamma \in S^{\prime}$ имеет р-адическое разложение $\gamma=$ $=\gamma_{0}+2 \gamma_{1}+\theta \gamma_{\theta}$. Тогда функция $\zeta(\gamma)$ принимает следующие значения:

$$
\zeta(\gamma)= \begin{cases}2^{m}-1, & \text { если } \gamma_{0}=0, \gamma_{1}=\gamma_{\theta}, \\ -1, & \text { если } \gamma_{0}=0, \gamma_{1} \neq \gamma_{\theta}, \\ -1 \pm 2^{s} \pm \omega 2^{s}, & \text { если } \gamma_{0} \neq 0, m=2 s+1 \text { нечетно } \\ -1 \pm 2^{s},-1 \pm \omega 2^{s}, & \text { если } \gamma_{0} \neq 0, m=2 s \text { четно }\end{cases}
$$

Доказательство. Пусть $\gamma_{0}=0$. Тогда $\gamma=2 \gamma_{1}+\theta \gamma_{\theta}$, и

$$
\begin{aligned}
\zeta(\gamma) & =\sum_{x \in \Gamma\left(S^{\prime}\right)^{*}} \chi\left(\operatorname{Tr}\left(2 \gamma_{1}+\theta \gamma_{\theta}\right) x\right)= \\
& =\sum_{x \in \Gamma\left(S^{\prime}\right)^{*}}(-1)^{\operatorname{tr}\left(\gamma_{1} x\right)}(-1)^{\operatorname{tr}\left(\gamma_{\theta} x\right)}=\sum_{x \in \Gamma\left(S^{\prime}\right)^{*}}(-1)^{\operatorname{tr}\left(\left(\gamma_{1}+\gamma_{\theta}\right) x\right)} .
\end{aligned}
$$


Если $\gamma_{1}+\gamma_{\theta}=0$, то сумма равна $\left|\Gamma\left(S^{\prime}\right)^{*}\right|=2^{m}-1$. Если же $\gamma_{1}+\gamma_{\theta} \neq 0$, то сумма равна -1 ввиду (13).

Пусть теперь $\gamma_{0} \neq 0$. По утверждению 2 имеем $|1+\zeta(\gamma)|^{2}=2^{m}$. Пусть $1+\zeta(\gamma)=a+b \omega$, где $a, b \in \mathbb{Z}$. Тогда

$$
|a+b \omega|^{2}=a^{2}+b^{2}=2^{m}, \quad a, b \in \mathbb{Z} .
$$

При $m=1$ все очевидно, поэтому будем считать, что $m \geq 2$. Числа $a, b$ одной четности. Если оба они нечетны, то $a^{2}+b^{2} \equiv 2(\bmod 4)$, чего не может быть. Значит, числа $a, b$ четные, $a=2 a_{1}, b=2 b_{1}, a_{1}^{2}+b_{1}^{2}=2^{m-2}$. Рассуждая аналогично, придем к уравнению $a_{s}^{2}+b_{s}^{2}=2^{m-2 s}$, где $a=2^{s} a_{s}$, $b=2^{s} b_{s}$. Тогда $a_{s}^{2}+b_{s}^{2}=2$, если $m$ нечетно, и $a_{s}^{2}+b_{s}^{2}=1$, если $m$ четно. Каждое из этих уравнений имеет 4 решения: $\left(a_{s}, b_{s}\right)=( \pm 1, \pm 1)$, если $m$ нечетно, и $\left(a_{s}, b_{s}\right)=( \pm 1,0),(0, \pm 1)$, если $m$ четно. Отсюда получаем, что $\zeta(\gamma)$ принимает указанные в формулировке теоремы значения.

Таким образом, сумма $\zeta(\gamma)$ принимает 6 различных значений. Теперь подсчитаем кратности, с которыми принимаются эти значения при всевозможных $\gamma \in S^{\prime}$. Рассмотрим сумму $\zeta(\gamma), \gamma \in S^{\prime}$, как отображение $\zeta: S^{\prime} \rightarrow \mathbb{C}$. Для $z \in \mathbb{C}$ обозначим

$$
N_{\zeta}(z)=\left|\zeta^{-1}(z)\right|=\left|\left\{\gamma \in S^{\prime}: \zeta(\gamma)=z\right\}\right| .
$$

Целое число $N_{\zeta}(z) \geq 0$ назовем кратностью значения $z$ функции $\zeta$. Вместо равенства $N_{\zeta}(z)=n$ говорят, что функция $\zeta(\gamma)$ принимает $n$ раз значение $z$ или что кратность значения $z$ равна $n$. Набор всех пар $\left(z, N_{\zeta}(z)\right), z \in \Gamma \zeta\left(S^{\prime}\right)$, называется распределением значений функции $\zeta(\gamma)$.

Теорема 4. Функиия $\zeta=\zeta(\gamma): S^{\prime} \rightarrow \mathbb{C}$ при $\gamma \in S^{\prime}$ имеет следующее распределение значений. Если $\gamma \notin\left(S^{\prime}\right)^{*}$, то

$$
\zeta(\gamma)= \begin{cases}2^{m}-1 & \left(2^{m} \text { раз }\right), \\ -1 & \left(2^{2 m}-2^{m} \text { раз }\right) .\end{cases}
$$

Если $\gamma \in\left(S^{\prime}\right)^{*}$, то при $m=2 s+1$ нечетном

$$
\zeta(\gamma)=\left\{\begin{array}{lll}
-1+2^{s} \pm w 2^{s} & \left(\left(2^{m}-1\right)\left(2^{4 s}+2^{3 s}\right)\right. & \text { раз }), \\
-1-2^{s} \pm w 2^{s} & \left(\left(2^{m}-1\right)\left(2^{4 s}-2^{3 s}\right)\right. & \text { раз }),
\end{array}\right.
$$

а при $m=2 s$ четном

$$
\zeta(\gamma)=\left\{\begin{array}{lll}
-1+2^{s} & \left(\left(2^{m}-1\right)\left(2^{4 s-2}+2^{3 s-1}\right)\right. & \text { раз }) \\
-1-2^{s} & \left(\left(2^{m}-1\right)\left(2^{4 s-2}-2^{3 s-1}\right)\right. & \text { раз }), \\
1 \pm w 2^{s} & \left(\left(2^{m}-1\right) \cdot 2^{4 s-2} \quad \text { раз }\right) &
\end{array}\right.
$$


Доказательство. Пусть $\gamma=\gamma_{0}+2 \gamma_{1}+\theta \gamma_{\theta} \in S^{\prime}$. По теореме 3 равенство $\zeta(\gamma)=2^{m}-1$ выполняется тогда и только тогда, когда $\gamma_{0}=0, \gamma_{1}=\gamma_{\theta}$. В кольце $S^{\prime}$ существует в точности $2^{m}$ таких значений $\gamma$.

Аналогично, $\zeta(\gamma)=-1$ тогда и только тогда, когда $\gamma_{0}=0, \gamma_{1} \neq \gamma_{\theta}$. Таких значений $\gamma$ в точности $2^{2 m}-2^{m}$. Соотношение (17) доказано.

Пусть теперь $\gamma \in\left(S^{\prime}\right)^{*}$. Тогда $\zeta(\gamma)$ принимает значения, перечисленные в теореме 3 , и эти значения указаны в (18), (19). Подсчитаем кратности этих значений.

Из определения (14) следует, что значения $\zeta(\gamma)$ одинаковы для всех $\gamma$, принадлежащих одному смежному классу группы $\left(S^{\prime}\right)^{*}$ по подгруппе $\Gamma\left(S^{\prime}\right)^{*}$. Пусть $H$ - некоторая полная система представителей смежных классов $\left(S^{\prime}\right)^{*}$ по $Г\left(S^{\prime}\right)^{*}$. Тогда

$$
|H|=\left|\left(S^{\prime}\right)^{*}\right| /\left|\Gamma\left(S^{\prime}\right)^{*}\right|=\left(2^{3 m}-2^{2 m}\right) /\left(2^{m}-1\right)=2^{2 m} .
$$

Кратности значений функции $\zeta(\gamma), \gamma \in\left(S^{\prime}\right)^{*}$, равны кратностям значений функции $\zeta(\gamma), \gamma \in H$, умноженным на $\left|\Gamma\left(S^{\prime}\right)^{*}\right|=2^{m}-1$.

Так как $(-1)^{2^{m}}=1 \neq-1$, то $-1 \notin \Gamma\left(S^{\prime}\right)$. Поэтому элементы $\gamma$ и $-\gamma$ представляют различные смежные классы $\left(S^{\prime}\right)^{*}$ по $\Gamma\left(S^{\prime}\right)^{*}$. Будем считать, что система представителей $H$ удовлетворяет условию

$$
\gamma \in H \quad \Rightarrow \quad-\gamma \in H \text {. }
$$

Пусть $m=2 s+1$. Рассмотрим разбиение множества $H$ на 4 подмножества в соответствии с тем, какие значения принимает функция $\zeta(\gamma)$ :

$$
H=H_{++} \cup H_{+-} \cup H_{-+} \cup H_{--},
$$

где

$$
H_{ \pm, \pm}=\left\{\gamma \in H: \zeta(\gamma)=-1 \pm 2^{s} \pm \omega 2^{s}\right\} .
$$

Из определения (14) следует, что $\zeta(-\gamma)=\bar{\zeta}(\gamma)$. Поэтому ввиду (20) $\left|H_{ \pm+}\right|=$ $=\left|H_{ \pm-}\right|$. Обозначим

$$
\left|H_{++}\right|=\left|H_{+-}\right|=M, \quad\left|H_{-+}\right|=\left|H_{--}\right|=N .
$$

Тогда $2 M+2 N=|H|=2^{2 m}=2^{4 s+2}$.

Применяя лемму 1в), находим:

$$
\sum_{\gamma \in H} \zeta(\gamma)=\sum_{\gamma \in H} \sum_{x \in \Gamma\left(S^{\prime}\right)^{*}} \chi(\operatorname{Tr}(\gamma x))=\sum_{y \in\left(S^{\prime}\right)^{*}} \chi(\operatorname{Tr}(y))=0 .
$$


Сумму в левой части можно разбить на четыре суммы по $\gamma \in H_{ \pm, \pm}$. В peзультате получим соотношение

$M\left(-1+2^{S}+\omega 2^{S}\right)+M\left(-1+2^{S}-\omega 2^{S}\right)+N\left(-1-2^{S}+\omega 2^{S}\right)+N\left(-1-2^{S}-\omega 2^{S}\right)=0$,

или $M+N=2^{s}(M-N)$. Решение системы из двух уравнений

$$
\left\{\begin{array}{l}
2 M+2 N=2^{4 s+2} \\
M+N=2^{s}(M-N),
\end{array}\right.
$$

имеет вид $M=2^{4 s}+2^{3 s}, N=2^{4 s}-2^{3 s}$. Это кратности значений функции $\zeta(\gamma)$ при $\gamma \in H$. Умножая их на $2^{m}-1$, получаем соотношение (18).

Пусть $m=2 s$. Рассуждая аналогично, обозначим

$$
\begin{aligned}
M & =\left|\left\{\gamma \in H: \zeta(\gamma)=-1+2^{s}\right\}\right|, \\
N & =\left|\left\{\gamma \in H: \zeta(\gamma)=-1-2^{s}\right\}\right|, \\
P & =\left|\left\{\gamma \in H: \zeta(\gamma)=-1 \pm \omega 2^{s}\right\}\right| .
\end{aligned}
$$

Тогда $M+N+2 P=|H|=2^{2 m}=2^{4 s}$. Разбивая равную нулю сумму (21) на четыре суммы, приходим к равенству

$$
M\left(-1+2^{s}\right)+N\left(-1-2^{s}\right)+P\left(-1+\omega 2^{s}\right)+P\left(-1-\omega 2^{s}\right)=0,
$$

откуда $M-N=2^{s}$. Чтобы найти третье уравнение относительно $M, N, P$, вычислим «второй момент»:

$$
\begin{aligned}
m_{2} & =\sum_{\gamma \in H} \zeta(\gamma)^{2}=\sum_{\gamma \in H}\left(\sum_{x \in \Gamma\left(S^{\prime}\right)^{*}} \chi(\operatorname{Tr}(\gamma x))\right)^{2}= \\
& =\sum_{\gamma \in H} \sum_{x, y \in \Gamma\left(S^{\prime}\right)^{*}} \chi(\operatorname{Tr}(\gamma x)) \chi(\operatorname{Tr}(\gamma y)) .
\end{aligned}
$$

Здесь

$$
\chi(\operatorname{Tr}(\gamma x)) \chi(\operatorname{Tr}(\gamma y))=\chi(\operatorname{Tr}(\gamma(x+y)))=\chi\left(\operatorname{Tr}\left(\gamma x\left(1+\frac{y}{x}\right)\right)\right) .
$$

Заменяя $y / x$ на $z \in \Gamma\left(S^{\prime}\right)^{*}$, получаем

$$
m_{2}=\sum_{\gamma \in H} \sum_{x, z \in \Gamma\left(S^{\prime}\right)^{*}} \chi(\operatorname{Tr}(\gamma x(1+z))) .
$$

2010, T. 1, № 4, C. 85-109 
Выделим слагаемое при $z=1$ :

$$
\begin{aligned}
m_{2} & =\sum_{\gamma \in H} \sum_{x \in \Gamma\left(S^{\prime}\right)^{*}} \chi(\operatorname{Tr}(2 \gamma x))+\sum_{\gamma \in H} \sum_{x \in \Gamma\left(S^{\prime}\right)^{*}} \sum_{z \in \Gamma\left(S^{\prime}\right)^{*} \backslash\{1\}} \chi(\operatorname{Tr}(\gamma x(1+z)))= \\
& =\sum_{x \in\left(S^{\prime}\right)^{*}} \chi(\operatorname{Tr}(2 x))+\sum_{z \in \Gamma\left(S^{\prime}\right)^{*} \backslash\{1\}} \sum_{x \in\left(S^{\prime}\right)^{*}} \chi(\operatorname{Tr}(x(1+z))) .
\end{aligned}
$$

В силу леммы 1в) вторая сумма равна 0 . Тогда

$$
\begin{aligned}
m_{2} & =\sum_{x \in\left(S^{\prime}\right)^{*}} \chi(\operatorname{Tr}(2 \gamma x))=\sum_{x \in\left(S^{\prime}\right)^{*}}(-1)^{\operatorname{tr}(\bar{\gamma} \bar{x})}= \\
& =2^{2 m} \sum_{\bar{x} \in\left(\bar{S}^{\prime}\right)^{*}}(-1)^{\operatorname{tr}(\bar{\gamma} \bar{x})}=2^{2 m} \cdot(-1)=-2^{2 m} .
\end{aligned}
$$

Возвращаясь к определению второго момента, разобьем сумму (22) на четыре суммы в соответствии с четырьмя значениями, принимаемыми функцией $\zeta(\gamma)$ :

$$
m_{2}=M\left(-1+2^{s}\right)^{2}+N\left(-1-2^{s}\right)^{2}+P\left(-1+\omega 2^{s}\right)^{2}+P\left(-1-\omega 2^{s}\right)^{2}=-2^{2 m},
$$

или после сокращений $M+N-2 P=0$. Решая систему из трех уравнений

$$
\left\{\begin{array}{l}
M+N+2 P=2^{4 s} \\
M-N=2^{3 s} \\
M+N-2 P=0
\end{array}\right.
$$

получаем $M=2^{4 s-2}+2^{3 s-1}, N=2^{4 s-2}-2^{3 s-1}, P=2^{4 s-2}$. Умножение этих кратностей значений функции $\zeta(\gamma), \gamma \in H$, на $2^{m}-1$ дает соотношения (19).

Следствие. Пусть $H$ - произвольная полная система представителей смежных классов группы $\left(S^{\prime}\right)^{*}$ по подгруппе $\Gamma\left(S^{\prime}\right)^{*}$, удовлетворяющая условию (20). Тогда функиия $\zeta(\gamma)$ при $\gamma \in H$ имеет следующее распределение значений: если $m=2 s+1$ нечетно, то

$$
\zeta(\gamma)=\left\{\begin{array}{lll}
-1+2^{s} \pm \omega 2^{s} & \left(2^{4 s}+2^{3 s}\right. & \text { раз }), \\
-1-2^{s} \pm \omega 2^{s} & \left(2^{4 s}-2^{3 s}\right. & \text { раз }),
\end{array}\right.
$$

и если $m=2$ s четно, то

$$
\zeta(\gamma)=\left\{\begin{array}{lll}
-1+2^{s} & \left(2^{4 s-2}+2^{3 s-1}\right. & \text { раз }) \\
-1-2^{s} & \left(2^{4 s-2}-2^{3 s-1} m\right. & \text { раз }) \\
-1 \pm \omega 2^{s} & \left(2^{4 s-2} \quad \text { раз }\right)
\end{array}\right.
$$




\section{6. Корреляционная функция семейства $A$}

Для $z \in \mathbb{C}$ обозначим

$$
N_{A}(z)=\left|\left\{(u, v, t): C_{u, v}(t)=z, u, v \in A, 0 \leq t<T\right\}\right| .
$$

Набор пар $\left(z, N_{A}(z)\right)$, где числа $z \in \mathbb{C}$ берутся такими, что $N_{A}(z)>0$, называется распределением значений кросс-корреляционной функции семейства $A$, а число $N_{A}(z)-$ кратностью значения $z$. Пользуясь найденными кратностями значений функции $\zeta(\gamma)$ и соотношениями (14), найдем кратности значений кросс-корреляционной функции $C_{u, v}(t)$ для последовательностей $u, v \in A$.

Теорема 5. Пусть $F(x) \in S[x]-$ отмеченный многочлен степени $m \geq 1$ и периода $T=2^{m}-1$, и пусть $A=L_{S}(F) \backslash 0$. Тогда функиия кросскорреляции $C_{u, v}(t), u, v \in A, 0 \leq t<T$, принимает следующие значения со следующиим кратностями:

$$
C_{u, v}(t)= \begin{cases}2^{m}-1 & \left(\left(2^{m}-1\right)\left(\left(2^{3 m}-2\right)\left(2^{m}+1\right)+1\right) \quad \text { раз }\right), \\ -1 & \left(\left(2^{m}-1\right)\left(2^{3 m}-2\right)\left(2^{2 m}-2^{m}\right) \quad \text { раз }\right),\end{cases}
$$

кроме того, если $m=2 s+1$ нечетно, то

$$
C_{u, v}(t)=\left\{\begin{array}{c}
-1+2^{s} \pm \omega 2^{s} \\
-1-2^{s} \pm \omega 2^{s}
\end{array} \quad\left(\begin{array}{ll}
\left(2^{m}-1\right)^{2}\left(2^{3 m}-2\right)\left(2^{4 s}+2^{3 s}\right) & \text { раз }), \\
\left(2^{m}-12^{2 m}-2\right)\left(2^{4 s}-2^{3 s}\right) & \text { раз }
\end{array}\right),\right.
$$

и если $m=2 s$ четно, то

$$
C_{u, v}(t)=\left\{\begin{array}{lll}
-1+2^{s} & \left(\left(2^{m}-1\right)^{2}\left(2^{3 m}-2\right)\left(2^{4 s-2}+2^{3 s-1}\right)\right. & \text { раз } \\
-1-2^{s} & \left(2^{m}-1\right)^{2}\left(2^{3 m}-2\right)\left(2^{4 s-2}-2^{3 s-1}\right) & \text { раз } \\
-1 \pm \omega 2^{s} & \left.\left(2^{m}-1\right)^{2}\left(2^{3 m}-2\right) \cdot 2^{4 s-2} \quad \text { раз }\right) .
\end{array}\right.
$$

Доказательство. Пусть $\alpha \in S^{\prime}$ - корень многочлена $F(x)$ и последовательности $u, u^{\prime} \in A$ имеют представления (15) функцией след. Согласно (16) $C_{u, u^{\prime}}(t)=\zeta(\gamma)$, где $\gamma=c-c^{\prime} \alpha^{t}$. Обозначим

$$
N(\gamma)=\left|\left\{\left(c, c^{\prime}, t\right): \gamma=c-c^{\prime} \alpha^{t}, c, c^{\prime} \in S^{\prime} \backslash 0,0 \leq t<T\right\}\right| .
$$

Тогда, очевидно,

$$
N_{A}(z)=\sum_{\gamma \in S^{\prime}, \zeta(\gamma)=z} N(\gamma)
$$

Найдем величину $N(\gamma)$ для фиксированного $\gamma \in S^{\prime}$. 
Условие $\gamma=c-c^{\prime} \alpha^{t}$ запишем в виде

$$
c=\gamma+c^{\prime} \alpha^{t}, \quad c, c^{\prime} \in S^{\prime} \backslash 0, \quad 0 \leq t<T,
$$

и рассмотрим его как уравнение относительно $c, c^{\prime}, t$. Пусть вначале $\gamma \neq 0$. Заметим, что для каждого $t$ существует единственное такое $c^{\prime} \in S^{\prime} \backslash 0$, что $\gamma+c^{\prime} \alpha^{t}=0$, и для этого $c^{\prime}$ уравнение (27) не имеет решений $c \in S^{\prime} \backslash 0$. Таких пар $\left(c^{\prime}, t\right)$ имеется $T$ штук. Для остальных пар $\left(c^{\prime}, t\right)$, число которых равно $\left(2^{3 m}-1\right) T-T=\left(2^{3 m}-2\right) T$, уравнение (27) имеет единственное решение $c$. Поэтому число решений

$$
N(\gamma)=\left(2^{3 m}-2\right) T=\left(2^{3 m}-2\right)\left(2^{m}-1\right), \quad \gamma \neq 0 .
$$

Если же $\gamma=0$, то для каждой пары $\left(c^{\prime}, t\right)$ существует единственное решение $c$, значит, число решений уравнения (27) равно числу пар $\left(c^{\prime}, t\right)$, т. е.

$$
N(0)=\left(2^{3 m}-1\right) T=\left(2^{3 m}-1\right)\left(2^{m}-1\right) .
$$

Теперь найдем сумму (26), подставив в нее найденные значения $N(\gamma)$. Согласно определению (14) $\zeta(0)=\left|\Gamma\left(S^{\prime}\right)^{*}\right|=2^{m}-1$. Поэтому в сумме (26) величина $N(0)$ присутствует только при $z=2^{m}-1$. В этом случае

$$
N_{A}\left(2^{m}-1\right)=\sum_{\gamma \in S^{\prime}, \zeta(\gamma)=2^{m}-1} N(\gamma)=N(0)+\sum_{\gamma \in S^{\prime} \backslash 0, \zeta(\gamma)=2^{m}-1}\left(2^{3 m}-2\right)\left(2^{m}-1\right) .
$$

По теореме 4 количество таких элементов $\gamma \in S^{\prime} \backslash 0$, что $\zeta(\gamma)=2^{m}-1$, равно $N_{\zeta}\left(2^{m}-1\right)=2^{m}$. Отсюда

$N_{A}\left(2^{m}-1\right)=N(0)+2^{m}\left(2^{3 m}-2\right)\left(2^{m}-1\right)=\left(2^{m}-1\right)\left(\left(2^{3 m}-2\right)\left(2^{m}+1\right)+1\right)$.

При $z \neq 2^{m}-1$ сумма (26) вычисляется проще:

$$
N_{A}(z)=\sum_{\gamma \in S^{\prime}, \zeta(\gamma)=z}\left(2^{3 m}-2\right)\left(2^{m}-1\right)=N_{\zeta}(z)\left(2^{3 m}-2\right)\left(2^{m}-1\right) .
$$

Остается воспользоваться значениями $N_{\zeta}(z)$, найденными в теореме 4 .

Следствие. Для кросс-корреляционной функции последовательностей из семейства А справедлива оиенка

$$
C_{\max } \leq 1+\sqrt{T+1} .
$$


Доказательство. Если $m=2 s+1$, то

$$
C_{\max } \leq \sqrt{\left(-1-2^{s}\right)^{2}+\left(2^{s}\right)^{2}}=\sqrt{2^{2 s+1}+2^{s+1}+1} \leq 1+\sqrt{2^{2 s+1}} .
$$

Если же $m=2 s$, то

$$
C_{\max } \leq 1+2^{s}=1+\sqrt{2^{2 s}}
$$

Так как $T+1=2^{m}$, то в обоих случаях получили требуемую оценку.

На практике используются семейства, которые не содержат последовательностей, являющихся сдвигами друг друга. Построим такое семейство на основе семейства $A$.

Пусть $\widetilde{A}$ - некоторое полное множество представителей сдвигов последовательностей семейства $A$. Так как $A$ содержит $2^{3 m}-1$ последовательностей периода $2^{m}-1$, то множество $\widetilde{A}$ содержит $\left(2^{3 m}-1\right) /\left(2^{m}-1\right)=2^{2 m}+$ $+2^{m}+1$ последовательностей. Понятно, что кросс-корреляционная функция последовательностей семейства $\widetilde{A}$ принимает те же значения, что и кросскорреляционная функция последовательностей семейства $A$. Ниже мы подсчитаем распределение этих значений для семейства $\widetilde{A}$, а также покажем, что это распределение не зависит от выбора представителей, образующих $\widetilde{A}$.

Занумеруем последовательности, входящие в семейство $A$, следующим образом:

$$
\widetilde{A}=\left\{u_{0}, u_{1}, \ldots, u_{2^{m}+2^{2 m}}\right\} .
$$

Будем считать, что последовательность $u_{n}$ имеет следующее представление функцией след:

$$
u_{n}(i)=\operatorname{Tr}\left(c_{n} \alpha^{i}\right), \quad i \geq 0, \quad 0 \leq n \leq 2^{m}+2^{2 m},
$$

где $c_{n} \in S^{\prime} \backslash 0$. Рассмотрим подробнее последовательности $u_{n}$ и коэффициенты $C_{n}$.

Пусть $u \in A$ - произвольная последовательность с представлением функцией след $u(i)=\operatorname{Tr}\left(c \alpha^{i}\right)$. Ее циклические сдвиги есть последовательности из $A$, у которых константа в представлении функцией след равна $c \alpha^{t}$, $0 \leq t<T$. Возможны два случая: $c \in J\left(S^{\prime}\right) \backslash 0$ или $c \in\left(S^{\prime}\right)^{*}$.

Если $c \in J\left(S^{\prime}\right) \backslash 0$, то $c=2 x+\theta y$, где $x, y \underset{\widetilde{A}}{y}\left(S^{\prime}\right)$, и $c \alpha^{t}=c x \alpha^{t}+c y \alpha^{t}$. Так как $\left|J\left(S^{\prime}\right) \backslash 0\right|=2^{2 m}-1$, то в множество $\widetilde{A}$ входит $\left(2^{2 m}-1\right) /\left(2^{m}-1\right)=$ $=2^{m}+1$ таких последовательностей. Одна из них имеет константу $c$ вида $c=2 x+\theta x$. Эту последовательность обозначим через $u_{0}$, а остальные $u_{1}, \ldots, u_{2^{m}}$. Множество соответствующих им констант обозначим:

$$
H_{0}=\left\{c_{0}, c_{1}, \ldots, c_{2^{m}}\right\} .
$$


Если же $c \in\left(S^{\prime}\right)^{*}$, то константы $c \alpha^{t}, 0 \leq t<T$, соответствующие сдвигам последовательности $u$, образуют смежный класс группы $\left(S^{\prime}\right)^{*}$ по подгруппе $\Gamma\left(S^{\prime}\right)^{*}$ с представителем $c$. Поэтому элементы $c_{2^{m}+1}, \ldots, c_{2^{m}+2^{2 m}}$ образуют полную систему представителей смежных классов группы $\left(S^{\prime}\right)^{*}$ по подгруппе $\Gamma\left(S^{\prime}\right)^{*}$. Обозначим эту систему

$$
H=\left\{c_{2^{m}+1}, \ldots, c_{2^{m}+2^{2 m}}\right\} .
$$

Пусть $H_{1}=H_{0} \cup H$. Окончательно можем записать:

$$
H_{1}=H_{0} \cup H=\left\{c_{0}, c_{1}, \ldots, c_{2^{m}}, c_{2^{m}+1}, \ldots, c_{2^{m}+2^{2 m}}\right\},
$$

где $c_{0}, c_{1}, \ldots, c_{2^{m}} \in J\left(S^{\prime}\right)$, причем $\gamma_{1}\left(c_{0}\right)=\gamma_{\theta}\left(c_{0}\right)$ и $\gamma_{1}\left(c_{i}\right) \neq \gamma_{\theta}\left(c_{i}\right)$ для $1 \leq i \leq$ $\leq 2^{m}$, и $c_{2^{m}+1}, \ldots, c_{2^{m}+2^{2 m}} \in\left(S^{\prime}\right)^{*}$.

Заметим, что если $c \in J\left(S^{\prime}\right)$, то $c=-c$. Если же $c \in\left(S^{\prime}\right)^{*}$, то элементы $c$ и $-c$ различны и лежат в разных смежных классах по подгруппе $\Gamma\left(S^{\prime}\right)^{*}$, поскольку $-1 \notin \Gamma\left(S^{\prime}\right)$. Для последовательностей из $A$ это означает, что если $u \in A$, то либо $u=-u$ (в случае, когда $u(i) \in J\left(S^{\prime}\right), i \geq 0$ ), либо последовательности $u,-u$ лежат на разных циклах. Поэтому семейство $\widetilde{A}$ можно выбрать так, чтобы выполнялось условие

$$
u \in \widetilde{A} \Rightarrow-u \in \widetilde{A} .
$$

Это условие равносильно тому, что множество $H$ удовлетворяет условию (20).

Теорема 6. Пусть $F(x) \in S[x]-$ отмеченный многочлен степени $m \geq 1$ и периода $T=2^{m}-1, A=L_{S}(F) \backslash 0$, и пусть $\widetilde{A}-$ некоторое полное множество представителей сдвигов последовательностей семейства $A$, удовлетворяющее условию (28). Тогда $\widetilde{A}$ состоит из $2^{2 m}+2^{m}+1$ последовательностей периода $T$, и функция кросс-корреляиии $C_{u, v}(t), u, v \in \widetilde{A}$, $0 \leq t<T$, принимает следующие значения со следующими кратностями:

$$
C_{u, v}(t)= \begin{cases}2^{m}-1 & \left(2^{3 m}+2^{2 m}+2^{m}-1 \quad \text { раз }\right), \\ -1 & \left(2^{m}\left(2^{3 m}-2\right) \quad \text { раз }\right),\end{cases}
$$

кроме того, если $m=2 s+1$ нечетно, то

$$
C_{u, v}=\left\{\begin{array}{lll}
-1+2^{s} \pm \omega 2^{s} & \left(\left(2^{3 m}-2\right)\left(2^{4 s}+2^{3 s}\right)\right. & \text { раз }), \\
-1-2^{s} \pm \omega 2^{s} & \left(\left(2^{3 m}-2\right)\left(2^{4 s}-2^{3 s}\right)\right. & \text { раз }),
\end{array}\right.
$$


а если $m=2$ четено, то

$$
C_{u, v}= \begin{cases}-1+2^{s} & \left(\left(2^{3 m}-2\right)\left(2^{4 s-2}+2^{3 s-1}\right) \text { раз }\right), \\ -1-2^{s} & \left(\left(2^{3 m}-2\right)\left(2^{4 s-2}-2^{3 s-1}\right) \text { раз }\right), \\ -1 \pm \omega 2^{s} & \left(\left(2^{3 m}-2\right) \cdot 2^{4 s-2} \text { раз }\right) .\end{cases}
$$

Доказательство. Для $z \in \mathbb{C}$ обозначим

$$
N_{\widetilde{A}}(z)=\left|\left\{\left(u_{i}, u_{j}, t\right): C_{u_{i}, u_{j}}(t)=z, u_{i}, u_{j} \in \widetilde{A}, 0 \leq t<T\right\}\right| .
$$

Наша цель заключается в том, чтобы найти $N_{\widetilde{A}}(z)$ для всех $z$. Согласно (16)

$$
C_{u_{i}, u_{j}}(t)=\zeta(\gamma), \quad \text { где } \gamma=c_{i}-c_{j} \alpha^{t} .
$$

Обозначим

$$
\widetilde{N}(\gamma)=\left|\left\{\left(c_{i}, c_{j}, t\right): \gamma=c_{i}-c_{j} \alpha^{t}, 0 \leq i, j \leq 2^{2 m}+2^{m}, 0 \leq t<T\right\}\right| .
$$

Тогда, очевидно,

$$
N_{\tilde{A}}(z)=\sum_{\gamma \in S^{\prime}, \zeta(\gamma)=z} \tilde{N}(\gamma)
$$

Найдем величину $\widetilde{N}(\gamma)$ для $\gamma \in S^{\prime}$.

Для заданного $\gamma$ рассмотрим уравнение $\gamma=c_{i}-c_{j} \alpha^{t}$ относительно неизвестных $(i, j, t)$, где $c_{i}, c_{j} \in H_{1}, 0 \leq t<T$. Согласно определению множества $H_{1}$ произведение $c_{j} \alpha^{t}$ пробегает по одному разу все элементы из $S^{\prime} \backslash 0$. Поэтому если $\gamma \notin H_{1}$, то $c_{i}-\gamma \neq 0$, и для каждого $i$ уравнение имеет единственное решение $(j, t)$. Общее число решений уравнения равно $2^{2 m}+2^{m}+1$.

Если же $\gamma \in H_{1}$, то уравнение имеет единственное решение $(j, t)$ для каждого $i$, за исключением того, для которого $\gamma=c_{i}$; в последнем случае решения нет. Общее число решений равно $2^{2 m}+2^{m}$. Таким образом,

$$
\widetilde{N}(\gamma)= \begin{cases}2^{2 m}+2^{m}+1, & \gamma \in S^{\prime} \backslash H_{1}, \\ 2^{2 m}+2^{m}, & \gamma \in H_{1} .\end{cases}
$$

Подставляя в (32), получаем

$$
\begin{aligned}
N_{\widetilde{A}}(z) & =\sum_{\gamma \in S^{\prime}, \zeta(\gamma)=z}\left(2^{2 m}+2^{m}+1\right)-\sum_{\gamma \in H_{1}, \zeta(\gamma)=z} 1= \\
& =\left(2^{2 m}+2^{m}+1\right) N_{\zeta}(z)-\left|\left\{\gamma \in H_{1}: \zeta(\gamma)=z\right\}\right| .
\end{aligned}
$$


Величина $N_{\zeta}(z)$ найдена в теореме 4 . Остается подсчитать величину

$$
N_{\zeta, H_{1}}(z)=\left|\left\{\gamma \in H_{1}: \zeta(\gamma)=z\right\}\right| \text {. }
$$

Это будет сделано с помощью теоремы 3.

Напомним, что множество $H_{1}=H_{0} \cup H$ содержит один элемент $c_{0} \in$ $\in J\left(S^{\prime}\right)$, у которого $\gamma_{1}\left(c_{0}\right)=\gamma_{\theta}\left(c_{0}\right)$, и $2^{m}$ элементов $c_{1}, \ldots, c_{2^{m}} \in J\left(S^{\prime}\right)$, у которых $\gamma_{1}\left(c_{i}\right) \neq \gamma_{\theta}\left(c_{i}\right)$. Остальные элементы из $H_{1}$ лежат в $\left(S^{\prime}\right)^{*}$. Отсюда по теореме 3 получаем, что

$$
N_{\zeta, H_{1}}\left(2^{m}-1\right)=1, \quad N_{\zeta, H_{1}}(-1)=2^{m} .
$$

Подставляя в (33) и учитывая, что по теореме 4 выполняются равенства $N_{\zeta}\left(2^{m}-1\right)=2^{m}$ и $N_{\zeta}(-1)=2^{2 m}-2^{m}$, находим

$$
\begin{aligned}
& N_{\widetilde{A}}\left(2^{m}-1\right)=\left(2^{2 m}+2^{m}+1\right) 2^{m}-1=2^{3 m}+2^{2 m}+2^{m}-1, \\
& N_{\widetilde{A}}(-1)=\left(2^{2 m}+2^{m}+1\right)\left(2^{2 m}-2^{m}\right)-2^{m}=2^{m}\left(2^{3 m}-2\right) .
\end{aligned}
$$

Соотношение (29) доказано.

Найдем теперь $N_{\zeta, H_{1}}(z)$ при $z \notin\left\{2^{m}-1,-1\right\}$. По теореме 3 тогда $\zeta(\gamma)=z$ только для $\gamma \in\left(S^{\prime}\right)^{*}$. Следовательно,

$$
N_{\zeta, H_{1}}(z)=N_{\zeta, H}(z)=|\{\gamma \in H: \zeta(\gamma)=z\}| .
$$

Эта величина найдена в следствии теоремы 4. В результате соотношение (33) принимает вид

$$
N_{\widetilde{A}}(z)=\left(2^{2 m}+2^{m}+1\right) N_{\zeta}(z)-N_{\zeta, H}(z),
$$

где величина $N_{\zeta}(z)$ найдена в теореме 4 , а величина $N_{\zeta, H}(z)-$ в следствии теоремы 4. Сравнивая теорему 4 и ее следствие, находим, что $N_{\zeta}(z)=\left(2^{m}-\right.$ $-1) N_{\zeta, H}(z)$, поэтому

$$
N_{\widetilde{A}}(z)=\left(\left(2^{2 m}+2^{m}+1\right)\left(2^{m}-1\right)-1\right) N_{\zeta, H}(z)=\left(2^{3 m}-2\right) N_{\zeta, H}(z) .
$$

Пользуясь теперь следствием теоремы 4, получаем соотношения (30) и (31). 


\section{Список литературы}

1. Кузьмин А.С., Куракин В.Л., Нечаев А. А. Статистические свойства линейных рекуррент над кольцами Галуа и квазифробениусовыми модулями характеристики 4. - В сб.: Труды по дискретной математике. Т. 4. - М.: ФИЗМАТЛИТ, 2001, c. 91-128.

2. Нечаев А.А., Кузьмин А.С., Куракин В.Л. Структурные, аналитические и статистические свойства линейных и полилинейных рекуррент. - В сб.: Труды по дискретной математике. Т. 3. - М.: ФИЗМАТЛИТ, 2000, с. 155-194.

3. Нечаев А.А., Кузьлин А.С., Куракин В. Л. Вполне равномерные линейные рекурренты над кольцами Галуа и QF-модулями характеристики 4. - В сб.: Труды по дискретной математике. Т. 5. - М.: ФИЗМАТЛИТ, 2002, с. 103-158.

4. Нечаев А. А. Код Кердока в циклической форме. - Дискретная математика, 1989, т. 1, вып. 4, с. 123-139.

5. Нечаев А.А. Конечные квазифробениусовы модули, приложения к кодам и линейным рекуррентам. - Фундам. и Прикл. Матем., 1995, т. 1, № 1, с. 229-254.

6. Сидельников В. М. О взаимной корреляции последовательностей. - Доклады АН CCCP, 1971, т. 196, c. 531-534.

7. Boztas S., Hammons R., Kumar P. V. 4-phase sequences with near-optimum correlation properties. - IEEE Trans. Inform. Theory, 1992, v. 38, № 3, p. 1101-1113.

8. McDonald B. R. Finite rings with identity. - New York, Dekker, 1974.

9. Nechaev A. A. Finite rings with applications. - Handbook of Algebra (M. Hazewinkel ed.), v. 5, 2008, Elsevier, p. 213-320.

10. Welch L. R. Lower bounds on the maximum cross correlation of signals. - IEEE Trans. Inform. Theory, 1974, v. 20, № 3, p. 397-399. 